\title{
A Parametric Study of the Choice of Binary Interaction Parameter and Equation of State for High Pressure Vapor-Liquid Equilibrium of Nitrogen - n-Dodecane Binary System
}

\author{
B. Balaji ${ }^{1}$, Vasudevan Raghavan ${ }^{2^{*}}$ and K. Ramamurthi ${ }^{3}$ \\ Department of Mechanical Engineering, Indian Institute of Technology Madras, India \\ ${ }^{1}$ balajinaresh@gmail.com, ${ }^{2}$ raghavan@iitm.ac.in, ${ }^{3}$ krmurthi@iitm.ac.in
}

\begin{abstract}
A parametric study of the effects of binary interaction parameter and real-gas equations of state on the high pressure vapor-liquid equilibrium of nitrogen-n-dodecane system was carried out. Different values of the binary interaction parameter reported in literature, including one which depends on temperature, were employed in different equations of state to predict the vapor-liquid equilibrium as a function of ambient pressure and temperature. The findings were compared against the available experimental values reported in literature. Constant values of binary interaction parameter, estimated based on temperature dependent values, are demonstrated to predict the experimentally observed vapor-liquid equilibrium values accurately. The Peng-Robinson equation of state and an average binary interaction parameter were demonstrated to predict the vapor-liquid equilibrium over a wide range of temperature and pressures for nitrogen-n-dodecane binary system.
\end{abstract}

Keywords: Vapor-liquid equilibria; high pressure; binary interaction parameter; equation of state; binary system.

\section{Introduction}

Combustion devices operating at high power densities, such as the high performance liquid propellant rockets and liquid fuelled combustors, operate at high values of pressures often exceeding the critical pressures. Kerosene is a widely used fuel in aerospace applications, including liquid propellant rockets. A regular alkane, n-dodecane, has been considered as a surrogate of kerosene, since its properties are close to that of kerosene. Often in the mathematical models, n-dodecane is employed to simulate the evaporation and combustion characteristics of kerosene fuelled systems. In this study, high pressure vapor-liquid equilibrium of nitrogen-n-dodecane system is investigated.

The non-ideal effects, solubility of ambient gas in liquid fuel droplet and variations in thermo-physical properties influence the Vapor-Liquid Equilibrium (VLE) and the evaporation of the liquid fuel and its combustion. Vaporliquid equilibria of several binary systems have been studied in the past. Intermediate- and high-temperature studies involving nitrogen and alkanes, ranging from butane up to decane (Akers, Kehn \& Kilgore, 1954; Lehigh \& McKetta, 1966; Peter \& Eicke 1970; Brunner, Peter \& Wenzel, 1974; Kalra, Robinson \& Besserer, 1977; Krishnan, Kalra \& Robinson, 1977; Kalra, Ng, Miranda \& Robinson, 1978; Figuiere, Hom, Laugier, Renon, Richon, \& Szwarc, 1980; Legret, Richon \& Renon, 1981; Cohen \& Richon, 1986) as well as mixtures with benzene (Lewis \& Luke. 1933; Miller \& Dodge, 1940; Lin, Kim \& Chao, 1981; de Leeuw, Poot, de Loos \& de Swaan Arons, 1989; García-Sánchez, Eliosa-Jiménez, Silva-Oliver, \& GodínezSilva, 2007; García-Sánchez, Eliosa-Jiménez, Silva-Oliver \& García-Flores, 2009; Eliosa-Jiménez, Silva-Oliver, García-Sánchez \& de Ita de la Torre, 2007; Eliosa-Jiménez, García-Sánchez, Silva-Oliver \& Macías-Salinas, 2009; Silva-Oliver, Eliosa-Jiménez, García-Sánchez \& Avendaño-Gómez, 2006, 2007) and toluene (Prausnitz \& Benson, 1959; Field Wilhelm, \& Battino, 1974; Richon,
Laugier \& Renon, 1992) have been investigated. There are also a few data reported for systems containing hydrocarbons heavier than n-decane (Azarnoosh \& McKetta, 1963; D'Avila, Kaul \& Prausnitz, 1976; Lin, Kim \& Chao, 1981; Llave \& Chung, 1988; de Leeuw, de Loos, Kooijman, \& de Swaan Arons, 1992; Tong, Gao, Robinson Jr. \& Gasem, 1999; Gao, Robinson Jr., \& Gasem, 1999). The vapor-liquid phase behavior of a binary mixture is predicted using a real-gas equation of state (EOS), along with proper mixing rules. The EOS utilizes an empirically calculated quantity termed as binary interaction parameter $\left(k_{i j}\right)$. For a given binary system, different values of this experimentally determined binary interaction parameters are reported (García-Sánchez, Jiménez-Eliosa, Oliver-Silva \& Román-Vázquez, 2004; Meng \& Duan, 2005). For instance, more than one binary interaction parameter value has been reported for nitrogen-n-dodecane binary system. Gao, Robinson Jr., \& Gasem (1999) reported different values of $k_{i j}$ to be employed in Peng Robinson EOS (PREOS) (Peng \& Robinson, 1976) and Soave Redlich Kwong EOS (SRK-EOS) (Soave, 1972), which were temperature dependent. They had also reported an average $k_{i j}$ value for each EOS. The phase equilibrium of $n$-dodecane in nitrogen environment, at various ambient pressures and temperatures, could be influenced by the different values of the binary interaction parameter used in the real-gas EOS. Curtis \& Farrell (1992) predicted vapor-liquid equilibrium of nitrogen-n-dodecane system using a $k_{i j}$ value of 0.11 in their model with PR EOS; however, the model validation was done with a different fuel.

A limited number of experimental data is available for nitrogen-n-dodecane for a moderate range of pressures and temperatures (Llave \& Chung, 1988; Gao, Robinson Jr., \& Gasem, 1999). However, a theoretical model to predict the same, after due validation with experimental values, especially for a wide range of ambient pressures and 
temperatures, is scarce. The objective of the present study is to develop and validate a theoretical model, for VLE of nitrogen-n-dodecane system for a wide range of ambient conditions. The model is first employed in studying the effects of using different binary interaction parameters reported in literature. Thereafter, different EOS are studied to determine the effect of the choice of EOS on the predictions. The $k_{i j}$ value and the EOS pair, which provide closer agreement with experimental results, are used in the model to predict the VLE of nitrogen-n-dodecane system.

\section{Description of the Theoretical Model}

The model is based on the procedure illustrated in Reid et al. (1987). Thermodynamic equilibrium is assumed to prevail at the interface. The $\mathrm{p}-\mathrm{v}-\mathrm{T}$ relationship is determined by a real-gas equation of state, such as, the one as given below.

$$
p=\frac{R T}{v-b}-\frac{a}{v^{2}+u b v+w b^{2}}
$$

The variances in the parameters, $a, b, u$ and $w$ for different types of EOS are listed in Table 1. The temperature and pressure at the liquid fuel surface are $T_{s}$ and $p_{s}$, respectively. For a system in thermodynamic equilibrium, the pressure, the temperature and the fugacity of each species in the liquid- and gas-phases, must be equal (Reid et al. (1987)):

$$
f_{i}^{v}=f_{i}^{l} \text { or } y_{i} \phi_{i}^{v}=x_{i} \phi_{i}^{l}
$$

The fugacity $(f)$ in each phase is calculated as a function of the fugacity coefficient $(\phi)$ and liquid- and gasphase mole fractions, $x_{i}$ and $y_{i}$ of the $i^{\text {th }}$ species. The fugacity coefficient is primarily a function of pressure, temperature and composition, and is given by

$$
R T \ln \left(\phi_{i}\right)=\int_{v}^{\infty}\left[\left(\frac{\partial p}{\partial n_{i}}\right)_{T, v, n_{j}}-\frac{R T}{v}\right] d v-R T \ln z
$$

The fugacity coefficient also depends on $n_{j}$, the number of moles of $j^{\text {th }}$ species and $z$, the compressibility factor. For a mixture involving $N$ components, the mixing rules suggested by Chueh \& Prausnitz (1967) are used to estimate the parametric constants in the EOS, and can be expressed as follows.

$$
\begin{aligned}
& a=\sum_{i=1}^{N} \sum_{j=1}^{N} y_{i} y_{j} a_{i j} \\
& b=\sum_{i=1}^{N} y_{i} b_{i} \\
& a_{i j}=\left(1-k_{i j}\right) \sqrt{a_{i i} a_{j j}}
\end{aligned}
$$

The binary interaction parameter, $k_{i j}$ is taken from literature (Curtis \& Farrell (1992), Gao, Robinson Jr., \& Gasem (1999)). The fugacity coefficient of the $i^{\text {th }}$ component in the liquid- or the gas-phase is evaluated using Eqs. (1)-(3), as given below.

$$
\begin{aligned}
\ln \phi_{i}= & \frac{b_{i}}{b R T}(p v-R T)-\ln \left(\frac{p}{R T}(v-b)\right) \\
& -\frac{a}{\sqrt{u^{2}-4 w} b R T}\left[\frac{2 \sum_{j=1,2} y_{j} a_{i j}}{a}-\frac{b_{i}}{b}\right] \\
& \times \ln \left(\frac{2 v+\left(u+\sqrt{u^{2}-4 w}\right) b}{2 v+\left(u-\sqrt{u^{2}-4 w}\right) b}\right)
\end{aligned}
$$

The equations of state have been rewritten as a cubic polynomial in molar specific volume. Out of the three roots obtained by iterative process, the minimum real root is considered as the liquid volume and the maximum real root is taken as the gas-volume of the component in the interface (Reid, Prausnitz \& Poling, 1987). Eqns (2) to (5) are solved iteratively using Newton-Raphson method to predict the gas- and liquid- phase equilibrium compositions. The convergence is monitored by checking the difference in the values of the previous and the current iteration value. If the difference is less than $10^{-05}$, then convergence is said to be attained.

\section{Results and Discussion}

Curtis \& Farrell (1992) used a value of $k_{i j}=0.11$, to be employed with PR-EOS. Gao et al. (1999) have reported temperature dependent $k_{i j}$ values, $k_{i j}(T)$ and an average value of $k_{i j}=0.2082$ and $k_{i j}=0.2226$ for PR-EOS and SRKEOS respectively. Figure 1 shows the influence of different $k_{i j}$ values with PR-EOS on the liquid-phase nitrogen mole fraction in a binary nitrogen-n-dodecane system as a function of ambient pressure, for three ambient temperatures. Both temperature dependent and average $k_{i j}$ values from Gao, Robinson Jr., \& Gasem (1999) and the $k_{i j}$ value from Curtis \& Farrell (1992) have been employed. The experimental data of Gao, Robinson Jr., \& Gasem (1999) are shown by symbols in Figure 1.

It is seen that the nitrogen mole fraction in the liquidphase increases as the ambient pressure increases as a result of increased solubility of ambient gases into the liquidphase at higher pressures. It also increases with an increase in the ambient temperature. Even though all $k_{i j}$ values predict the trends quite well, it is observed that both temperature dependent and average $k_{i j}$ values (Gao, Robinson Jr., \& Gasem, 1999) predict the liquid-phase nitrogen mole fraction more accurately against the experimental data (Gao, Robinson Jr., \& Gasem, 1999), when compared to that predicted using the $k_{i j}$ value reported in Curtis \& Farrell (1992). A comparison between theoretical model predictions of the mole fraction of $n$ dodecane in the liquid-phase against the experimental data of Llave \& Chung (1988) is shown in Figure 2. As the ambient pressure increases, the liquid-phase mole fraction of n-dodecane decreases due to additional quantities of dissolved ambient gas in the liquid-phase. Unlike in Figure 1 , the deviation between the theoretical predictions using $k_{i j}$ $=0.11$ (Curtis \& Farrell, 1992) and the experimental data for liquid-phase $\mathrm{n}$-dodecane mole fraction prediction is much smaller. 


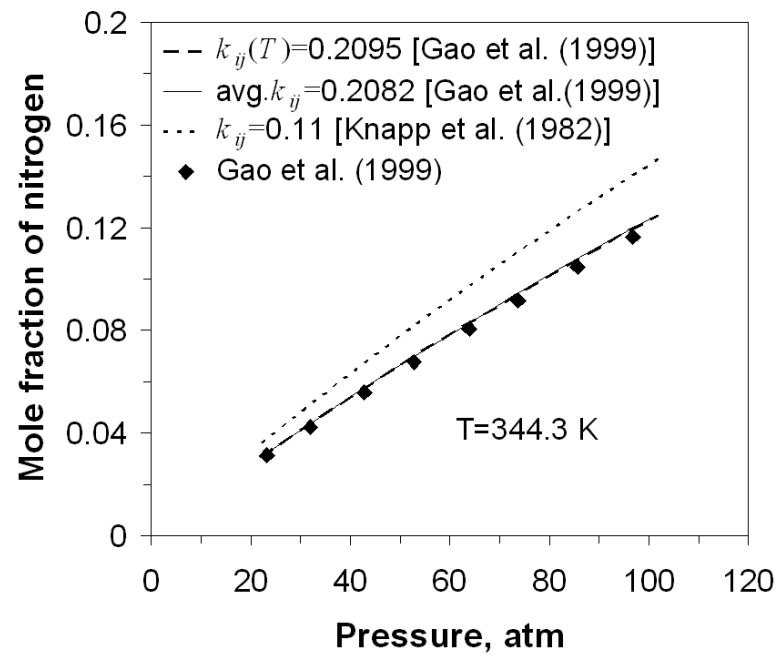

(a)

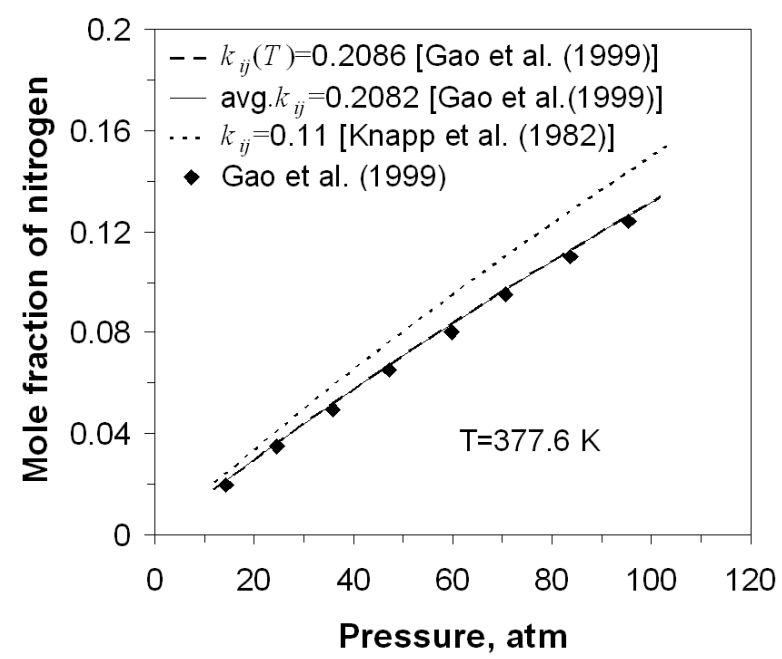

(b)

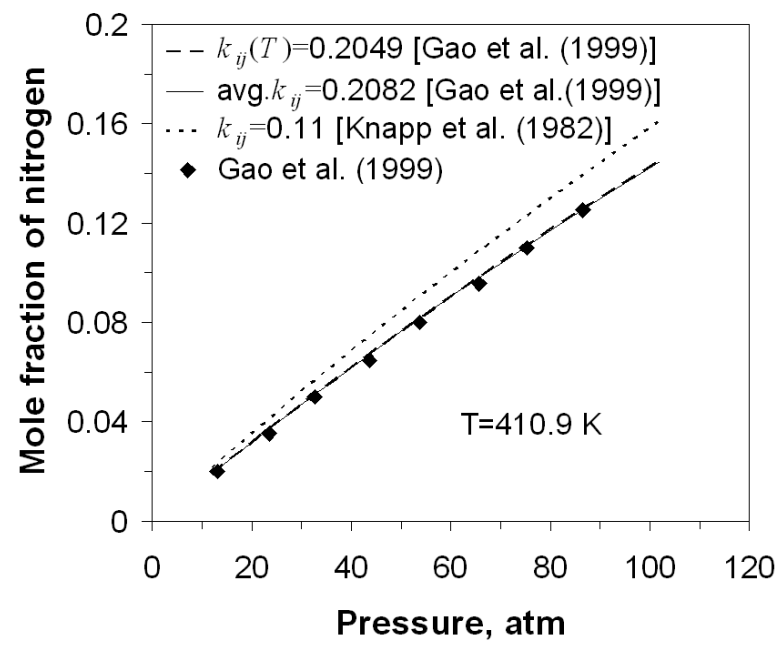

(c)

Figure 1: Liquid-phase mole fraction of nitrogen predicted by PR-EOS using binary interaction parameter $\left(k_{i j}\right)$ value reported by Knapp et al. (1982), temperature dependent and average $k_{i j}$ values reported by Gao et al. (1999) for a nitrogen-n-dodecane binary system in thermodynamic equilibrium at different temperatures (a) $344.3 \mathrm{~K}$ (b) 377.6 $K$ and (c) $410.9 \mathrm{~K}$.

In this case also, the temperature dependent and average $k_{i j}$ values of Gao, Robinson Jr., \& Gasem (1999) provide much better predictions. In all the cases considered in
Figures 1 and 2, it is seen that, theoretical predictions using the average $k_{i j}$ value are almost the same as obtained with the temperature dependent $k_{i j}$ values.

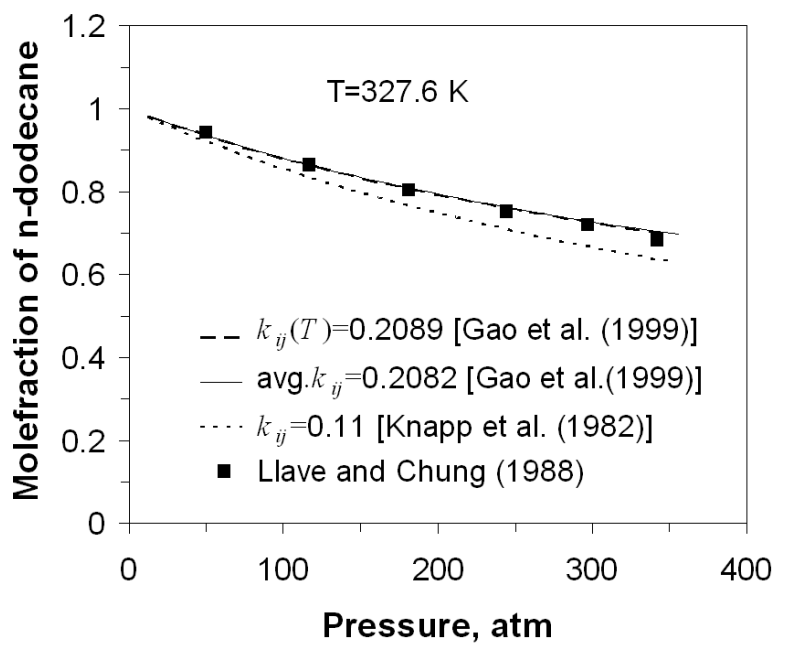

(a)

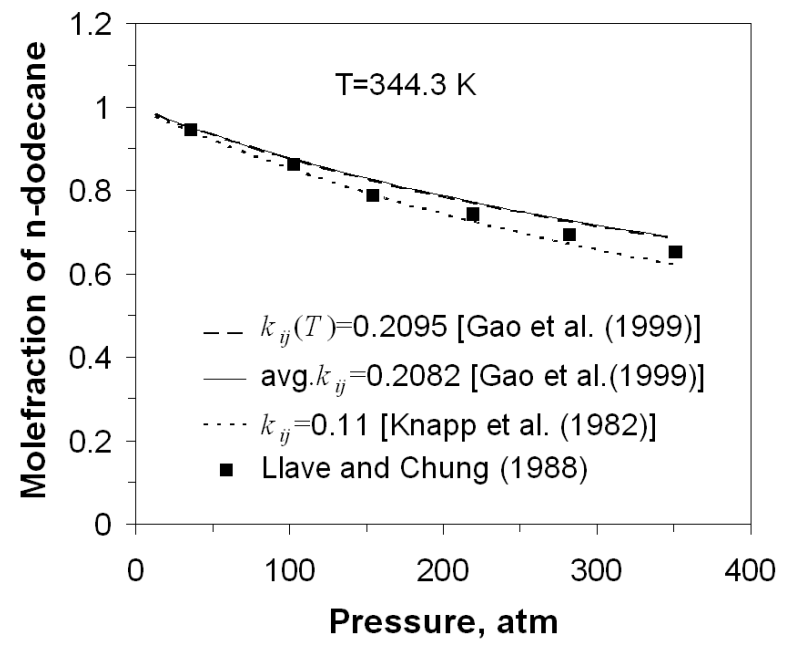

(b)

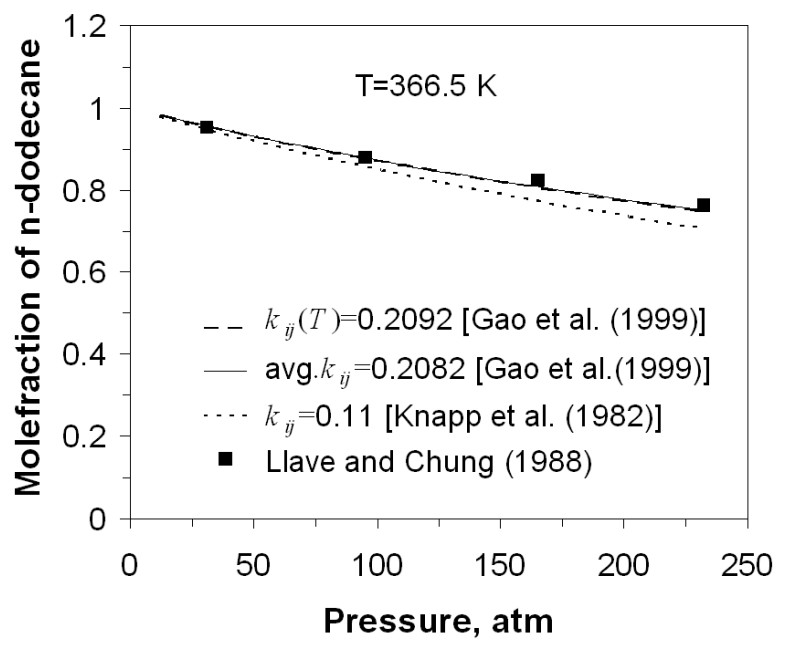

(c)

Figure 2: Liquid-phase mole fraction of n-dodecane predicted by PR-EOS using binary interaction parameter ( $\left.k_{i j}\right)$ value reported by Knapp et al. (1982), temperature dependent and average $k_{i j}$ values reported by Gao et al. (1999) for a nitrogen-n-dodecane binary system in thermodynamic equilibrium at different temperatures (a) $327.6 \mathrm{~K}$ (b) $344.3 \mathrm{~K}$ and (c) $366.5 \mathrm{~K}$. 
Therefore, subsequently the average $k_{i j}$ values are used to calculate the phase equilibrium. The percentage deviations between the experimental data and the theoretical predictions using different $k_{i j}$ values are shown in Table 2, which consolidates all the above results. The predictions using different EOS such as RK-EOS, SRKEOS and PR-EOS are compared with the experimental data reported in Gao, Robinson Jr., \& Gasem (1999) and Llave \& Chung (1988) in Figures 3 and 4.

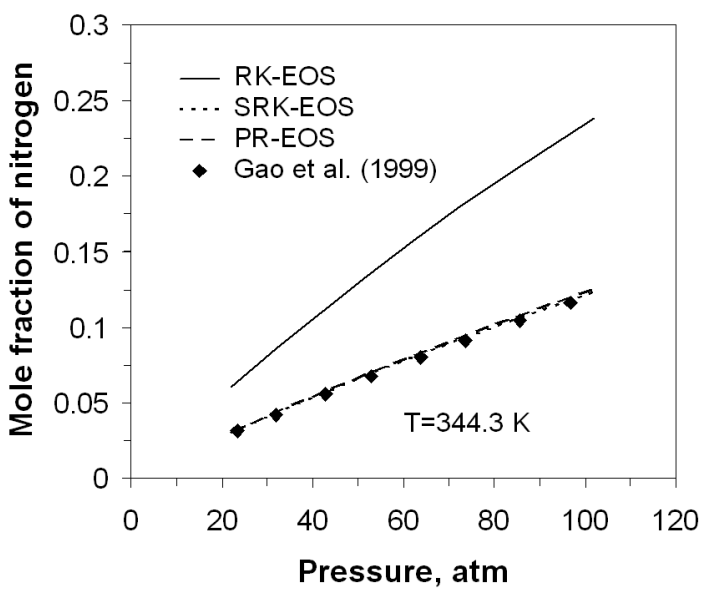

(a)

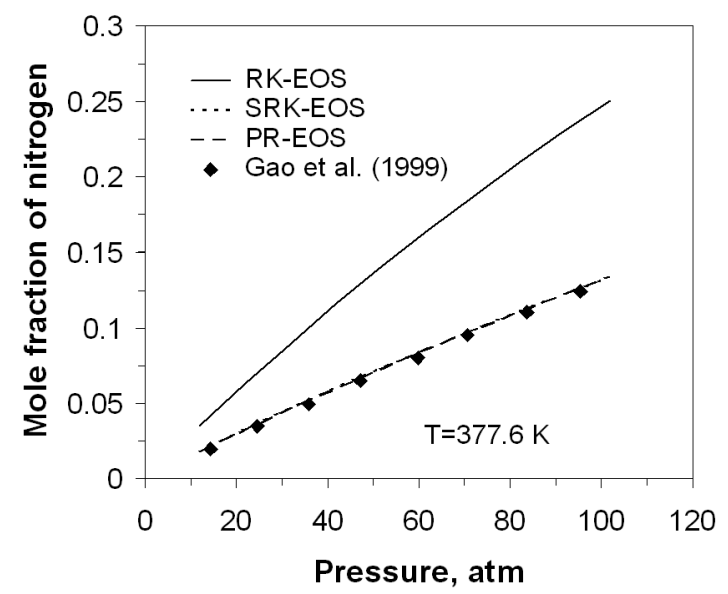

(b)

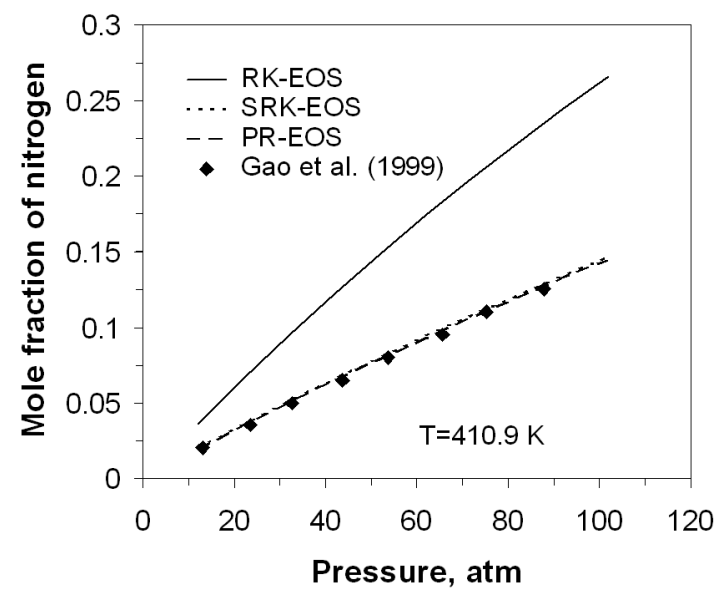

(c)

Figure 3: Liquid-phase mole fraction of nitrogen predicted by RK-EOS, SRK-EOS and PR-EOS using the average binary interaction parameter $\left(k_{i j}\right)$ value reported by Gao et al. (1999) for a nitrogen-n-dodecane binary system in thermodynamic equilibrium at different temperatures (a) $344.3 \mathrm{~K}$ (b) $377.6 \mathrm{~K}$ and (c) $410.9 \mathrm{~K}$.

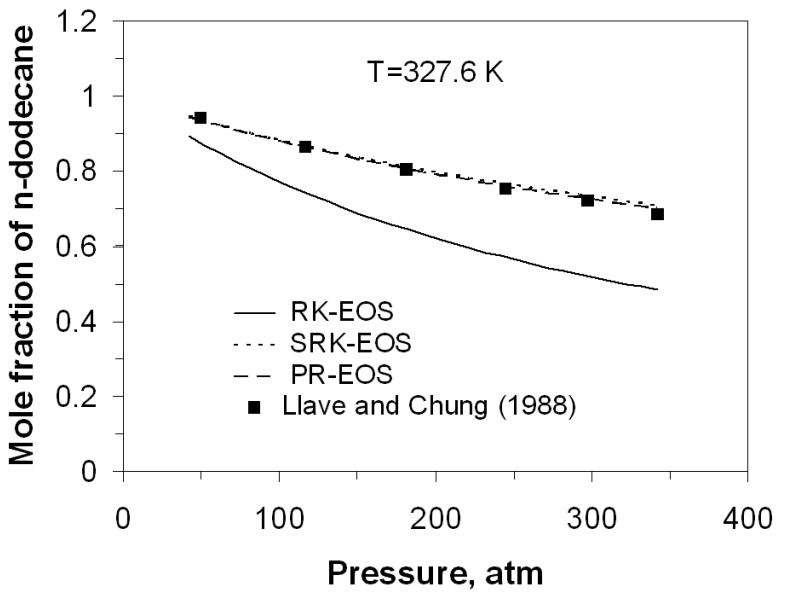

(a)

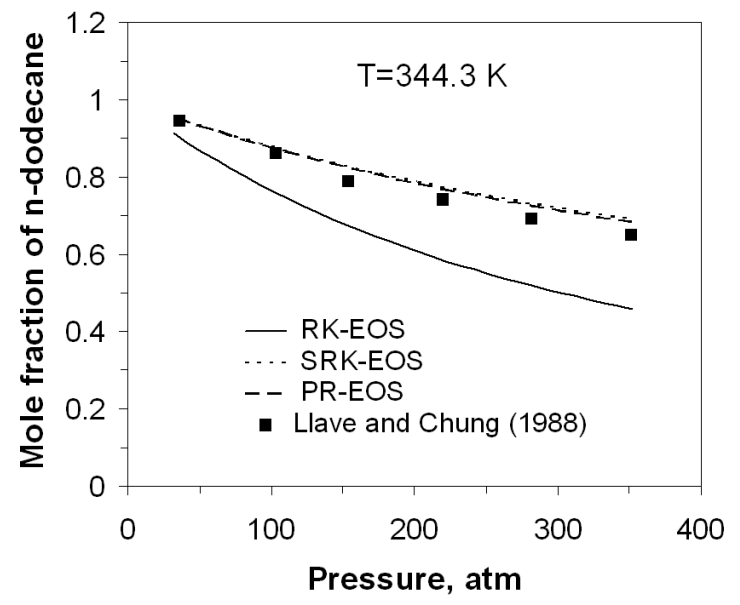

(b)

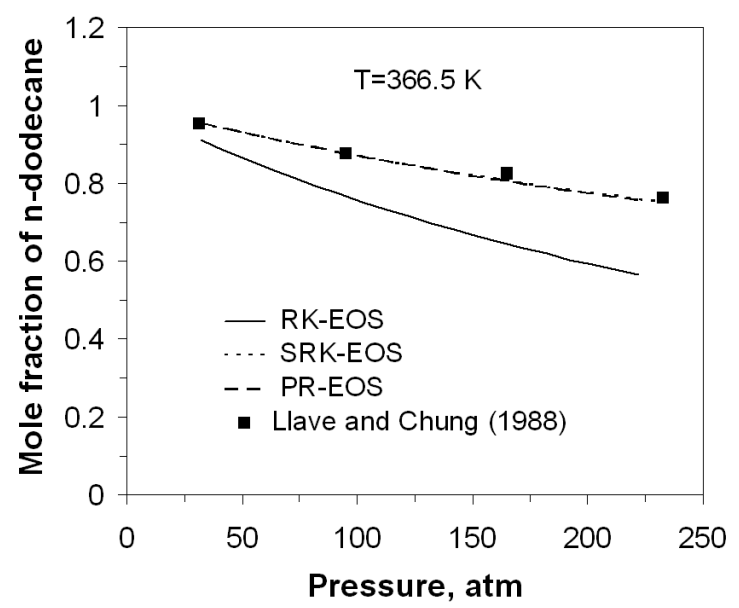

(c)

Figure 4: Liquid-phase mole fraction of $n$-dodecane predicted by RK-EOS, SRK-EOS and PR-EOS using average binary interaction parameter $\left(k_{i j}\right)$ value reported by Gao et al. (1999) for a nitrogen-n-dodecane binary system in thermodynamic equilibrium at different temperatures (a) $327.6 \mathrm{~K}$ (b) $344.3 \mathrm{~K}$ and (c) $366.5 \mathrm{~K}$.

The average $k_{i j}$ value reported for PR-EOS and SRKEOS in Gao, Robinson Jr., \& Gasem (1999) has been employed. For RK-EOS, the average $k_{i j}$ value reported for SRK-EOS has been employed. It is seen from Figures 3 and 4 that the predictions from SRK-EOS and PR-EOS agree well with the experimental data and the predictions from RK-EOS deviate significantly. The deviation in the latter case is seen to increase with pressure as well. The average 
and maximum percentage errors between the experimental data and the theoretical predictions using different EOS are reported in Table 3. It is seen that in majority of the cases, the performance of PR-EOS is better than SRK-EOS.

The vapor- and liquid-phase mole fractions of $n$ dodecane in a nitrogen-n-dodecane binary system, as predicted by RK-EOS, SRK-EOS and PR-EOS, are shown in Figure 5. The right side curves show the variation of liquid-phase mole fractions of n-dodecane, while, the left side curves show its vapor-phase mole fractions. The theoretical predictions involving SRK-EOS and PR-EOS are almost similar. On the other hand, the predictions using RK-EOS show a significant deviation as compared to those of SRK-EOS and PR-EOS. The RK-EOS over predicts the vapor phase mole fraction of n-dodecane and liquid-phase solubility of nitrogen for all ambient conditions. For a given mixture composition and pressure, there is a critical mixing point, where the intensive properties of vapor- and liquidphases will become equal and the latent heat of vaporization value becomes zero. The critical mixing point is seen to decrease as the ambient pressure increases and is clearly shown in Figure 6. Both SRK-EOS and PR-EOS predict almost same values of the critical mixing points for the pressures of 150 and $200 \mathrm{~atm}$. The critical point temperature is under-predicted by RK-EOS; it is lower than those predicted by SRK-EOS and PR-EOS, by around $6.4 \%$ for $150 \mathrm{~atm}$ and around $8.5 \%$ for 200 atm, respectively. Also shown in Figure 6 is the boiling line, which indicates the maximum temperature value for a given pressure attained by the binary system in phase equilibrium.

The results indicate that the predictions using PR-EOS with an average $k_{i j}$ value of 0.2082 are able to match the experimental measurements quite accurately. Therefore, the pair of PR-EOS with $k_{i j}=0.2082$ has been employed to predict the vapor-liquid equilibrium of nitrogen-ndodecane binary system. Figure 7 shows the VLE in terms of the equilibrium ratio, $\mathrm{K}$, as a function of pressure at various temperatures. The line $K=1$ shows the locus of critical mixing points. Curves above the $K=1$ line shows the variation of the equilibrium ratio for nitrogen at different isotherms and the curves below show the same for $n-$ dodecane.

\section{Conclusions}

A theoretical study investigating the effects of binary interaction parameter and real-gas equation of state (EOS) on the prediction of high pressure vapor-liquid equilibrium quantities of nitrogen-n-dodecane system is presented. Different values of the binary interaction parameter reported in literature, including one which depends on temperature, have been employed in different equations of state comprising Peng-Robinson EOS, Soave Redlich Kwong EOS and Redlich Kwong EOS, to predict the vapor-liquid equilibrium as a function of ambient pressure and temperature. The theoretical results are compared against the available experimental values reported in literature. Average value of binary interaction parameter, estimated from temperature dependent values, is demonstrated to predict accurately the experimental values of VLE. Peng-Robinson EOS is seen to predict the VLE values much closer to the experimental results than other EOS. The Peng-Robinson equation of state and the average binary interaction parameter can be employed to predict the VLE of nitrogen-n-dodecane binary system over a wide range of temperatures and pressures.

\section{Nomenclature}

$a, b \quad$ parameters in equation of state

$f_{i} \quad$ fugacity of $i^{\text {th }}$ species

$k_{i j} \quad$ binary interaction parameter

$\mathrm{K} \quad$ equilibrium ratio $=y / x$

$m$ function of acentric factor

$n_{i} \quad$ number of moles of $i^{\text {th }}$ species

$N$ number of components in mixture

$p \quad$ pressure

$R \quad$ universal gas constant

$T$ temperature

$u, w \quad$ constants

$v \quad$ specific volume of the mixture

$x \quad$ liquid phase mole fraction

$y \quad$ vapor phase mole fraction

$z \quad$ compressibility factor

Greek symbols

$\alpha \quad$ temperature dependent function

$\omega \quad$ acentric factor

$\phi_{i} \quad$ fugacity coefficient of $i^{\text {th }}$ species

Subscripts

c critical property

$i \quad i^{\text {th }}$ species

$r \quad$ reduced property

$s \quad$ surface

Superscripts

$l \quad$ liquid phase

$v \quad$ vapor phase

\section{References}

Akers, W.W., Kehn, D.M., and Kilgore, C.H. (1954). Volumetric and phase behavior of nitrogen-hydrocarbon systems: nitrogen-n-heptane system. Ind. Eng. Chem., 46, 2536-2539.

Azarnoosh, A. and McKetta, J.J. (1963). Nitrogen-n-decane system in the two-phase region. J. Chem. Eng. Data, 8, 494-496.

Brunner, G., Peter, S., and Wenzel, H. (1974). Phase equilibrium in the systems n-heptane-nitrogen, methylcyclohexane-nitrogen and n-heptanemethylcycloheptane-nitrogen at high pressures. Chem. Eng. J., 7, 99-104.

Chueh, P.L. and Prausnitz, J.M. (1967). Vapor-liquid equilibria at high pressures: vapor phase fugacity coefficients in nonpolar and quantum-gas mixtures. Ind. Eng. Chem. Fundam., 6, 492-498.

Cohen, A. and Richon, D. (1986). A new apparatus for simultaneous determination of phase equilibria and rheological properties of fluids at high pressures: its application to coal pastes studies up to $773 \mathrm{~K}$ and $30 \mathrm{MPa}$. Rev. Sci. Instrum. 57, 1192-1195.

Curtis, E.W. and Farrell, P.V. (1992). A numerical study of high-pressure droplet vaporization. Combust. Flame, 90, 85-102.

D'Avila, S.G. Kaul, B.K. and Prausnitz, J.M. (1976). Solubilities of heavy hydrocarbons in compressed methane and nitrogen. J. Chem. Eng. Data, 21, 488-491.

de Leeuw, V.V., Poot, W., de Loos, Th.W. and de Swaan Arons, J. (1989). High pressure phase equilibria of the binary systems $\mathrm{N}_{2}+$ benzene, $\mathrm{N}_{2}+$ p-xylene and $\mathrm{N}_{2}+$ naphthalene. Fluid Phase Equilibria, 49, 75-101. 
de Leeuw, V.V., de Loos, Th.W., Kooijman, H.A., de Swaan Arons, J. (1992). The experimental determination and modeling of VLE for binary subsystems of the quaternary system $\mathrm{N}_{2}+\mathrm{CH}_{4}+\mathrm{C}_{4} \mathrm{H}_{10}$ $+\mathrm{C}_{14} \mathrm{H}_{30}$ up to 1000 bar and $440 \mathrm{~K}$. Fluid Phase Equilibria, 73, 285-321.

Eliosa-Jiménez, G., Silva-Oliver, G., García-Sánchez, F. and de Ita de la Torre A. (2007). High-pressure vaporliquid equilibria in the nitrogen $+\mathrm{n}$-hexane system. $J$. Chem. Eng. Data, 52, 395-404.

Eliosa-Jiménez, G., García-Sánchez, F., Silva-Oliver, G. and Macías-Salinas, R. (2009). Vapor-liquid equilibrium data for the nitrogen + n-octane system from (344.5 to 543.5$) \mathrm{K}$ and at pressures up to $50 \mathrm{MPa}$. Fluid Phase Equilibria, 282, 3-10.

Field, L.R., Wilhelm, E. and Battino, R. (1974). The solubility of gases in liquids. 6 . The solubility of $\mathrm{O}_{2}, \mathrm{~N}_{2}$, $\mathrm{CH}_{4}, \mathrm{CO}, \mathrm{CO}_{2}$ and $\mathrm{CF}_{4}$ in toluene and methylcyclohexane", 10 to $40^{\circ} \mathrm{C}$. J. Chem. Thermodynamics, 6, 237-243.

Figuiere, P., Hom, J.F., Laugier, S., Renon, H., Richon, D., and Szwarc, H. (1980). Vapor-liquid equilibria up to $40000 \mathrm{kPa}$ and $400^{\circ} \mathrm{C}$ : a new static method. AIChE J., $26,872-875$.

Gao, W. Robinson Jr. R.L., Gasem, K.A.M. (1999). Highpressure solubilities of hydrogen, nitrogen, and carbon monoxide in dodecane from 344 to $410 \mathrm{~K}$ at pressures to $13.2 \mathrm{MPa}$. J. Chem. Eng. Data, 44, 130-132.

Kalra, H., Ng, H.J., Miranda, R.D., and Robinson, D.B. (1978). Equilibrium phase properties of the nitrogenisobutane system. J. Chem. Eng. Data, 23, 321-324.

Kalra, H., Robinson, D.B., and Besserer, G.J. (1977). The equilibrium-phase properties of the nitrogen- $n$-pen tane system. J. Chem. Eng. Data, 22, 215-218.

Krishnan, T.R., Kalra, H. and Robinson, D.B. (1977). The equilibrium-phase properties of the nitrogen-isopentane system. J. Chem. Eng. Data, 22, 282-285.

Legret, D., Richon, D. and Renon, H. (1981). Vapor-liquid equilibria up to $100 \mathrm{MPa}$ : a new apparatus. AIChE J., 27, 203-207.

Lehigh, W.R. and McKetta, J.J. (1966) Vapor-liquid equilibrium in the ethane-n-butane-nitrogen system. $J$. Chem. Eng. Data, 11, 180-182.

Lewis, W.K. and Luke, C.D. (1933). Vapor-liquid equilibria of hydrocarbons at high pressures. Ind. Eng. Chem. 25, 725-727.

Lin, H.M., Kim, H., and Chao, K.C. (1981). Gas-liquid equilibria in nitrogen $+n$-hexadecane mixtures at elevated temperatures and pressures. Fluid Phase Equilibria, 7, 181-185.

Llave, F.M. and Chung, L.H. (1988). Vapor-liquid equilibria of nitrogen-hydrocarbon systems at elevated pressures. J. Chem. Eng. Data, 33, 123-128.

Meng, L. and Duan, Y.-Y. (2005). Prediction of the second cross virial coefficients of nonpolar binary mixtures. Fluid Phase Equilibria, 238, 229-238.
Miller, P. and Dodge, B.F. (1940). The system benzenenitrogen, liquid-vapor phase equilibria at elevated pressures. Ind. Eng. Chem., 32, 434-438.

Peng, D.Y. and Robinson, D.B. (1976) A new two-constant equation of state. Ind. Eng. Chem. Fundam., 15, 59-64.

Peter, S., and Eicke, H.F. (1970). Phase equilibrium in the systems nitrogen-n-heptane, nitrogen-2,2,4trimethylpentane, and nitrogen-methylcyclohexane at higher pressures and temperatures. Ber. Bunsen-Ges. 74, 190-194.

Prausnitz, J.M., and Benson, P.R. (1959) Solubility of liquids in compressed hydrogen, nitrogen, and carbon dioxide. AIChE J., 5, 161-164.

Redlich, O. and Kwong, J.N.S. (1949). On the Thermodynamics of Solutions. V. An equation of state. Fugacities of gaseous solutions. Chem. Rev., 44, 233244.

Reid, R.C., Prausnitz, J.M. and Poling, B.E. (1987). The properties of gases and liquids", $4^{\text {th }}$ ed., McGraw-Hill, New York.

Richon, D., Laugier, S. and Renon, H. (1992). Highpressure vapor-liquid equilibrium data for binary mixtures containing molecular nitrogen, carbon dioxide, hydrogen sulfide and an aromatic hydrocarbon or propylcyclohexane in the range 313-473 K. J. Chem. Eng. Data, 37, 264-268.

García-Sánchez, F., Jiménez-Eliosa, G., Oliver-Silva, G. and Román-Vázquez, R. (2004). Vapor-liquid equilibria of nitrogen-hydrocarbon systems using the PC-SAFT equation of state. Fluid Phase Equilibria, 217, 241-253.

García-Sánchez, F., Eliosa-Jiménez, G., Silva-Oliver, G. and Godínez-Silva, A. (2007). High-pressure (vapor + liquid) equilibria in the (nitrogen $+n$-heptane) system. J. Chem. Thermodynamics, 39, 893-905.

García-Sánchez, F., Eliosa-Jiménez, G., Silva-Oliver, G. and García-Flores, B.E. (2009). Vapor-liquid equilibrium data for the nitrogen $+\mathrm{n}$-decane system from (344 to 563 ) $\mathrm{K}$ and at pressures up to $50 \mathrm{MPa} . J$. Chem. Eng.Data, 54, 1560-1568.

Soave, G. (1972). Equilibrium constants from a modified Redlich-Kwong equation of state. Chem. Eng. Sci., 27, 1197-1203.

Silva-Oliver, G., Eliosa-Jiménez, G., García-Sánchez, F. and Avendaño-Gómez, J.R. (2006). High-pressure vapor-liquid equilibria in the nitrogen-n-pentane system. Fluid Phase Equilibria, 250, 50-61.

Silva-Oliver, G., Eliosa-Jiménez, G., García-Sánchez, F. and Avendaño-Gómez, J.R. (2007). High-pressure vapor-liquid equilibria in the nitrogen-n-nonane system. J. Supercrit. Fluids, 42, 36-47.

Tong, J., Gao, W., Robinson Jr. R.L., Gasem, K.A.M. (1999). Solubilities of nitrogen in heavy normal paraffins from 323 to $423 \mathrm{~K}$ at Pressures to $18.0 \mathrm{MPa}$. $J$. Chem. Eng. Data, 44, 784-787. 
Table 1: Parameters involved in different real-gas equations of state expressions

\begin{tabular}{ll}
\hline \hline \multicolumn{1}{c}{ EOS } & \multicolumn{1}{c}{ Parameters } \\
$\begin{array}{l}\text { Redlich-Kwong (RK) } \\
(u=1 \text { and } w=0)\end{array}$ & $a=0.42747 \frac{R^{2} T_{c}^{2.5}}{p_{c}} \alpha(T), b=0.08664 \frac{R T_{c}}{p_{c}}$ \\
\hline $\begin{array}{l}\text { Soave-Redlich-Kwong (SRK) } \\
(u=1 \text { and } w=0)\end{array}$ & $\alpha(T)=1 / T^{0.5}$ \\
& $\alpha\left(T_{r}\right)=\left[1+m(\omega)\left(1-T_{r}^{0.5}\right)\right]^{2}$ \\
& $m(\omega)=0.480+1.574 \omega-0.176 \omega^{2}$ \\
$\begin{array}{l}\text { Peng-Robinson (PR) } \\
(u=2 \text { and } w=-1)\end{array}$ & $a=0.45724 \frac{R^{2} T_{c}^{2}}{p_{c}} \alpha\left(T_{r}\right), b=0.0778 \frac{R T_{c}}{p_{c}}$ \\
\hline \hline
\end{tabular}

Table 2: Percentage deviations between the theoretical predictions using PR-EOS and the experimental data of Llave and Chung(1988), Gao et al. (1999), when different $k_{i j}$ values are employed.

\begin{tabular}{|c|c|c|c|c|c|c|}
\hline \multirow[t]{3}{*}{$T(\mathrm{~K})$} & \multicolumn{3}{|c|}{$\%$ Max. absolute error } & \multicolumn{3}{|c|}{$\%$ Average absolute error } \\
\hline & \multicolumn{6}{|c|}{ Liquid-phase mole fraction of n-dodecane } \\
\hline & $k_{i j}=0.11$ & $k_{i j}=k_{i j}(T)$ & $k_{i j}=0.2082$ & $k_{i j}=0.11$ & $k_{i j}=k_{i j}(T)$ & $k_{i j}=0.2082$ \\
\hline 327.6 & 7.227 & 2.654 & 2.593 & 5.290 & 1.229 & 1.191 \\
\hline 344.3 & 4.467 & 5.223 & 5.107 & 2.291 & 3.687 & 3.617 \\
\hline \multirow[t]{3}{*}{366.5} & 7.403 & 2.205 & 2.243 & 4.746 & 1.338 & 1.366 \\
\hline & \multicolumn{6}{|c|}{ Liquid-phase mole fraction of nitrogen } \\
\hline & $k_{i j}=0.11$ & $k_{i j}=k_{i j}(T)$ & $k_{i j}=0.2082$ & $k_{i j}=0.11$ & $k_{i j}=k_{i j}(T)$ & $k_{i j}=0.2082$ \\
\hline 344.3 & 22.443 & 3.245 & 3.476 & 21.954 & 2.810 & 3.041 \\
\hline 377.6 & 24.987 & 8.820 & 8.881 & 19.768 & 4.143 & 4.202 \\
\hline 410.9 & 17.083 & 4.785 & 4.382 & 15.251 & 3.058 & 2.657 \\
\hline
\end{tabular}

Table 3: Percentage deviations between the theoretical predictions using corresponding average $k_{i j}$ values reported in Gao et al. (1999) and the experimental data of Llave and Chung(1988), Gao et al. (1999), when different equations of state are employed.

\begin{tabular}{ccccccc}
\hline \hline$T(\mathrm{~K})$ & \multicolumn{1}{c}{ \% Max. absolute error } & \multicolumn{3}{c}{ \% Average absolute error } \\
\hline \hline \multicolumn{5}{c}{ Liquid-phase mole fraction of n-dodecane } \\
\cline { 2 - 7 } & PR & SRK & RK & PR & SRK & RK \\
\hline 327.6 & 2.593 & 3.849 & 29.176 & 1.191 & 1.917 & 21.647 \\
344.3 & 5.107 & 6.307 & 29.446 & 3.617 & 4.250 & 19.418 \\
366.5 & 2.243 & 2.034 & 27.494 & 1.366 & 1.184 & 18.713 \\
\hline \multicolumn{7}{c}{ Liquid-phase mole fraction of nitrogen } \\
\cline { 2 - 7 } & PR & SRK & RK & PR & SRK & RK \\
\hline 344.3 & 3.476 & 2.304 & 103.298 & 3.041 & 1.850 & 100.537 \\
377.6 & 8.881 & 9.645 & 112.067 & 4.202 & 4.609 & 100.303 \\
410.9 & 4.382 & 6.530 & 97.979 & 2.657 & 4.440 & 92.986 \\
\hline \hline
\end{tabular}



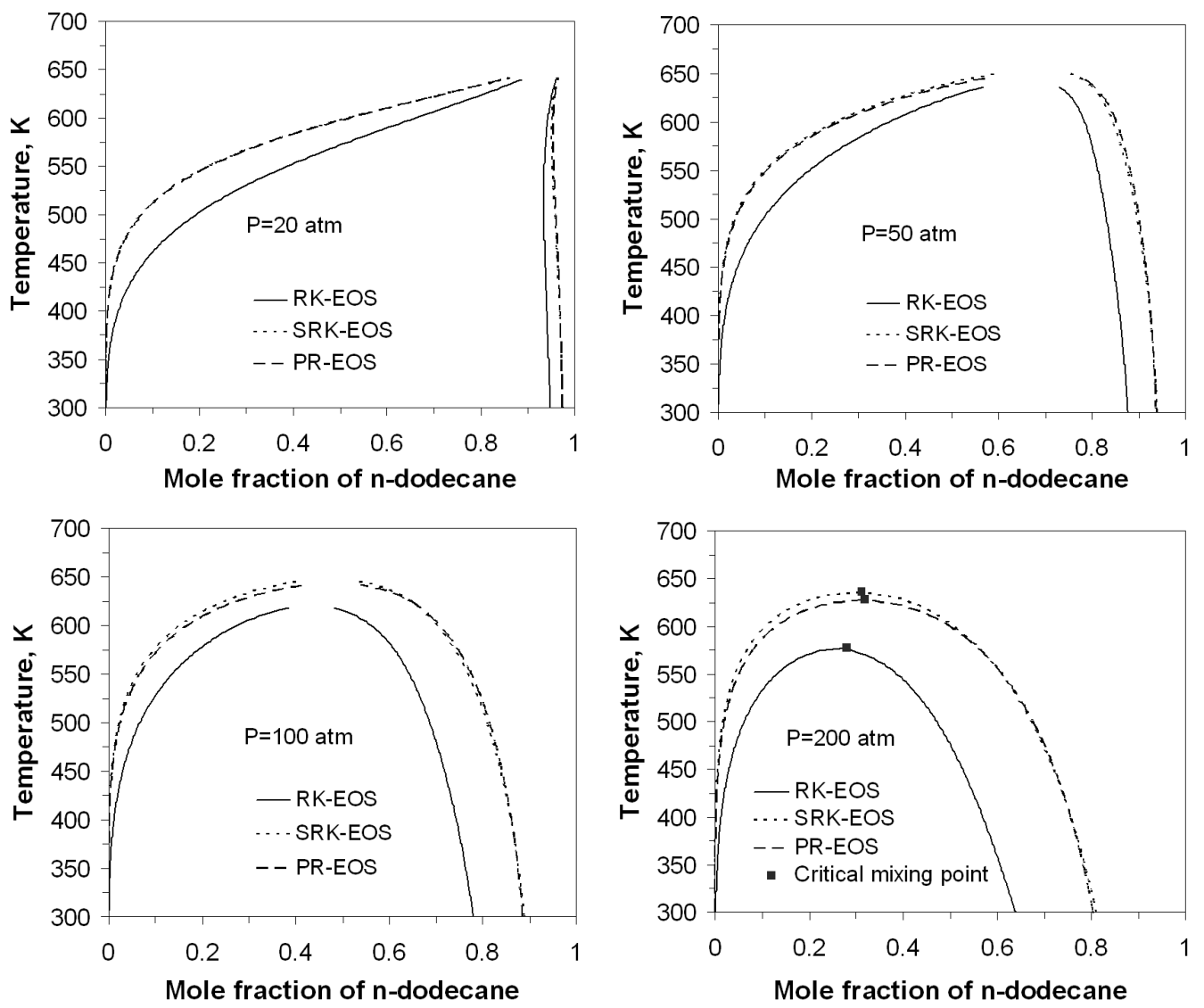

Figure 5: Mole fraction of n-dodecane in both phases, predicted by RK-EOS, SRK-EOS and PR-EOS using corresponding average binary interaction parameter $\left(k_{i j}\right)$ values reported by Gao et al. (1999) for a nitrogen-n-dodecane binary system, at several ambient pressures.

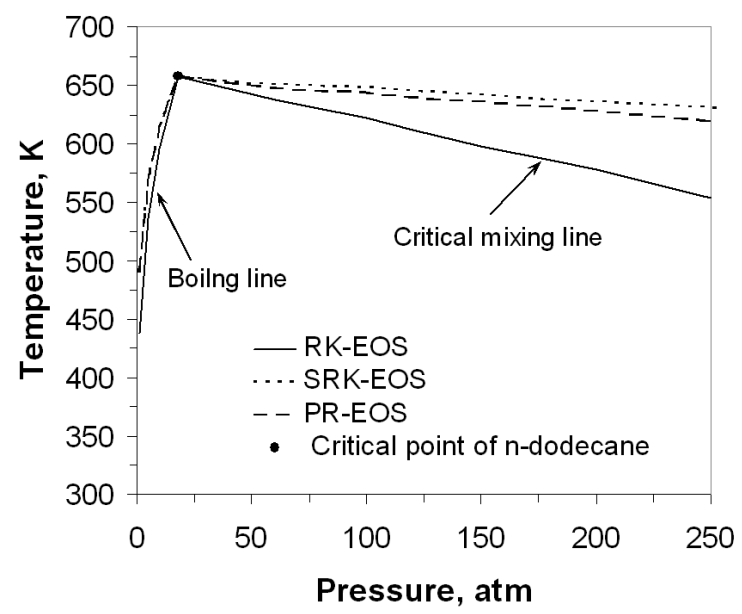

Figure 6: Critical locus of RK-EOS, SRK-EOS and PREOS using corresponding average binary interaction parameter $\left(k_{i j}\right)$ value reported by Gao et al. (1999) for a nitrogen-n-dodecane binary system.

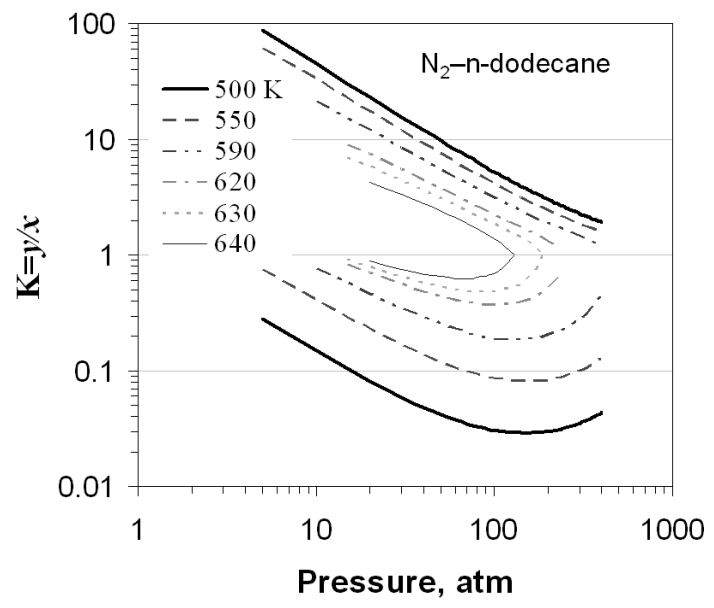

Figure 7: Effect of pressure on vapor liquid equilibrium ratio $(K)$ predicted by $P R$ EOS using corresponding average binary interaction parameter $\left(k_{i j}\right)$ value reported by Gao et al. (1999) for a nitrogen-n-dodecane binary system. 